\title{
Simulation of small size divertor tokamak plasma edge under effect of toroidal magnetic field reversal
}

\author{
Amr H. Bekheit \\ Plasma \& Nuclear Fusion department, Nuclear Research Centre, Atomic Energy Authority, Cairo, Egypt; \\ amrbekheitga@yahoo.com
}

Received 3 February 2011; revised 26 March 2011; accepted 6 April 2011.

\begin{abstract}
Asymmetries between the divertor legs of small size divertor (SSD) tokamak plasma edge are noticed to reverse when the direction of toroidal magnetic field is reversed. In the present paper the small size divertor tokamak plasma edge under effect of toroidal magnetic field reversal is simulated by B2SOLPS0.5.2D fluid transport code. The simulation demonstrate the following results: 1) Parallel (toroidal) flow flux and Mach number up to 0.6 at higher plasma density reverse with reverse toroidal magnetic direction in the edge plasma of small size divertor tokamak. 2) The radial electric field is toroidal magnetic direction independence in edge plasma of small size divertor tokamak. 3) For normal and reverse toroidal magnetic field, the strong ITB is located between the positions of the maximum and minimum values of the radial electric field shear. 4) Simulation result shows that, the structure of radial electric field at high field side (HFS) and low field side (LFS) is different. This difference result from the change in the parallel flux flows in the scrape off layer (SOL) to plasma core through separatrix. 5) At a region of strong radial electric field shear, a large reduction of poloidal rotation was observed. 6) The poloidal rotation is toroidal magnetic field direction dependence.
\end{abstract}

Keywords: Reverse Toroidal Field; B2SOLPS0.5.2D Code; ITB

\section{INTRODUCTION}

As a general trend, in single null divertor plasma, the in-out asymmetries in power and particle profile have long been observed between the divertor legs [1]. These asymmetries disappear or change significantly when the main toroidal magnetic field $B_{T}$ is reversed [2,3]. Reversal the direction of the toroidal magnetic field has been shown to alter these asymmetries and is accompanied by changes in the amount and location of radiation [2]. Also the direction of toroidal magnetic field determines the direction of plasma particles drifts. Therefore, the asymmetries that are sensitive to the field direction are almost certainly a reflection of the drifts [2]. In the experiment, in-out asymmetries show strong variance with discharge parameters, and in particular with the direction of toroidal magnetic field, $B_{T}$ reversal can help in establishing the physical mechanisms discriminate in their effect on the asymmetries between ion and electron drifts sides rather than between inner and outer sides [2]. The reversal of $B_{T}$ exchanges the sides, while the reversal of IP (poloidal current) leaves than unchanged. In this paper, we focus on the study of the of the reverse toroidal magnetic field direction on the edge plasma of small size divertor tokamak using 2-D SOLPS0.5 fluid transport code $[1,4]$; including the calculation of the radial electric field $E_{r}$. The basic item of "small size divertor tokamak (SSDT)" is stainless-steel discharge vessel consisting of two toroidal segments insulated from each other and sealed off by an O-ring. The chamber has rectangular cross-section $25 \mathrm{~cm}$ by $20 \mathrm{~cm}$. Six large lateral ports and 12 smaller windows at top and bottom of the vessel allow a good optical access to the entire plasma cross-section. The 180 toroidal field coils TF are directly glued on the vessel by epoxy resin. This makes dismantling and reconstruction of the tokamak very easy (1 day). The computation region for simulation of small size divertor tokamak is based on SN (Single Null) magnetic divertor and covers the SOL (Scrip Off Layer), core and private regions as shown in Figure 1. In computation region the coordinate which vary in the direction along flux surfaces ( $x$-coordinate or poloidal coordinate) and the coordinate which vary in the direction across flux surfaces ( $y$-coordinate or radial coordinate). The computation mesh is divided into $24 \times 96$ units (where $-1 \leq x \leq 96,-1 \leq y \leq 24$ ) and the separatrix was 


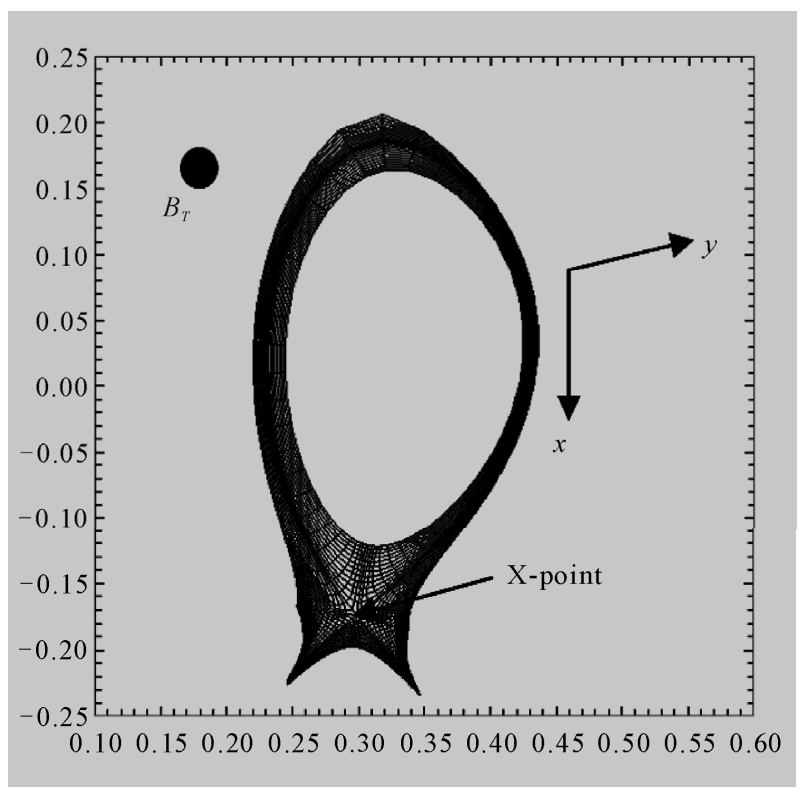

Figure 1. Coordinate system and simulation mesh: $x$ is the poloidal coordinate, $y$ is the radial coordinate orthogonal to the flux surfaces. The directions of magnetic field and plasma current correspond to normal operation of conditions of SSD tokamak ( $\nabla \mathrm{B}$ drift of ions directed towards the $\mathrm{x}$-point).

at $y=12$. The simulations were performed for L-regimes of SSDT (minor radius a $=0.1 \mathrm{~m}$, major radius $\mathrm{R}=0.3$ $\mathrm{m}, \mathrm{I}=50 \mathrm{kA}, B_{T}=1.7 \mathrm{~T}$, electron density at equatorial mid-plane $n_{e}=n_{i}=n=4 \times 10^{19} \mathrm{~m}^{-3}$, temperature heating $\mathrm{Ti}=6.9 \mathrm{~K} \mathrm{eV}$ ). The small size divertor tokamak has a lot of capabilities to study carefully cleaning and conditioning procedures. A fast and unproblematic exchange of magnetic divertors with different materials is possible and their influence on the plasma state can be examined. Most of the diagnostic techniques valuable for investigation of large tokamaks are applicable on small size tokamaks. Careful measurements of averaged plasma quantities may serve to improve the theoretical description of transport processes in the plasma bulk, in the SOL, the wall and divertor plates. The small size divertor (SSD) tokamak has a lot of application to study the following subjects [5]:

1) Study of plasma-surface interaction processes.

2) Compatibility tests of divertor material and plasma.

3) Influence of wall conditioning (Carbonisation, boronisation, siliconisation etc)

4) Fluctuation measurements, in particular by probes

5) Study the transport processes in plasma (transport theory )

6) Study spectroscopic analysis of impurities transport in plasma

7) Study the physics of edge plasma

8) Study the transition from low to high confinement (L-H) transition
9) Study the behavior of neutral particles near wall and divertor plates

10) Study the Material testing

11) The SSD tokomak experiment provides the possibility to study equilibrium, and the (resistive) MHD behavior of low-beta tokamak plasmas.

12) It will provide a very good opportunity in the future for students and young scientists to become acquainted with modern techniques the physics underlying the fusion research.

The present paper demonstrated that, the radial electric field of small size divertor tokamak independent on the toroidal magnetic field direction .The structure of the radial electric field at higher field side (HFS) and low field side (LFS) are discussed in this paper.

\section{THE MAIN SIMULATION RESULTS}

The B2.SOLPES0.5.2D code is used for present modeling. The code employed to solve the full two dimensional problem of the SOL multifluid transport Equations [4] uses an edge geometry and input assumptions for plasma transport based on a comparison with experimental data from other tokamaks. Multifluid plasmas consist of neutral particles, ions and electrons with various physical processes (e.g. ionization, recombination and charge exchange) [4].

The simulations were performed with B2SOLPS0.5.2D fluid transport code. As in similar codes the set of modified Braginski equations was solved [1-4]. The philosophy B2SOLPS0.5.2D fluid transport code (and other codes) is that the values of perpendicular transport coefficients are chosen to fit experimentally observed density, temperature radial profiles, density and temperature near the divertor plates. In the simulation presented below the perpendicular transport coefficients are replaced by the anomalous values: diffusion, electron, ion heat flux and perpendicular viscosity coefficients [1-4]. The perpendicular (anomalous) viscosity coefficient was taken in the form $\eta=n \cdot m i \cdot D$. At the inner boundary flux surface, which was located few $\mathrm{cm}$ from the separatrix, the density; the electron, ion heat fluxes and the average toroidal momentum flux were specified [1-4]. The boundary heat fluxes were imposed independently from the toroidal momentum flux thus providing the opportunity to investigate the dependence of radial electric field on these parameters [1-4]. For small size divertor tokamak operation, the simple anomalous cross-field transport is characterized by a particle diffusivity $D^{(\mathrm{AN})}=0.5 \mathrm{~m}^{2} / \mathrm{s}$, electron and ion heat diffusivities $\chi_{e, i}=0.7 \mathrm{~m}^{2} / \mathrm{s}$. The parallel heat and momentum transport are classical but flux limited. The computational region for modeling is based on SN (Single null) and covers the outer SOL and the divertor below the midplane plus a small segment of 
the region of closed flux surfaces and the private flux region(see Figure 1). The boundary conditions for modeling are given (1-4). The case of unbalance neutral beam injection is considered in this simulation. The main results of simulation are:

1) Typical profile of the radial electric field and ion temperature in both toroidal magnetic field directions (normal and reverse toroidal magnetic field) are presents in Figure 2, 3. In this case the radial electric field and radial ion temperature distribution are almost independent of the direction of toroidal magnetic field $B_{T}$.

2) Parallel (toroidal) flow flux is reverse with reverse toroidal magnetic field direction, with Mach number ( $M_{\| i}=V_{\| i} / C_{s}$, where Cs is sound speed) up to 0.6 in the SOL as shown in Figures 4, 5. Figures 4, 5 shows also the toroidal (parallel) flow flux is toroidal magnetic field $B_{T}$-dependence.

3) The structure of the radial electric at (HFS) and

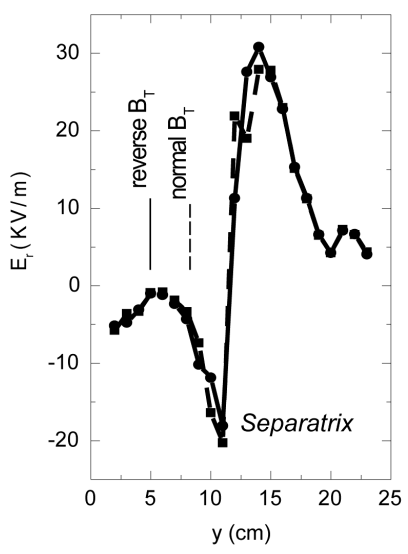

Figure 2. The radial distribution of radial electric field at normal and reverse toroidal magnetic field.

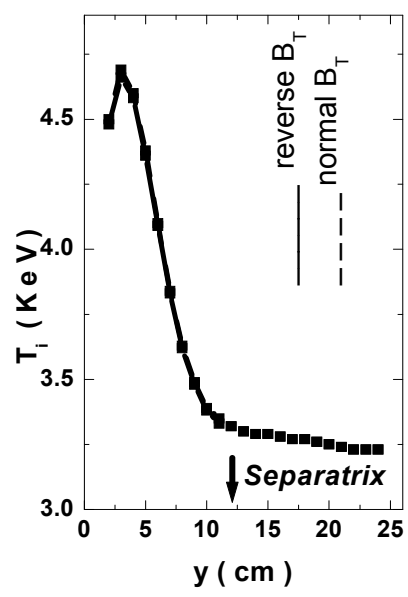

Figure 3. The radial distribution of ion temperature at normal and reverse toroidal magnetic field.

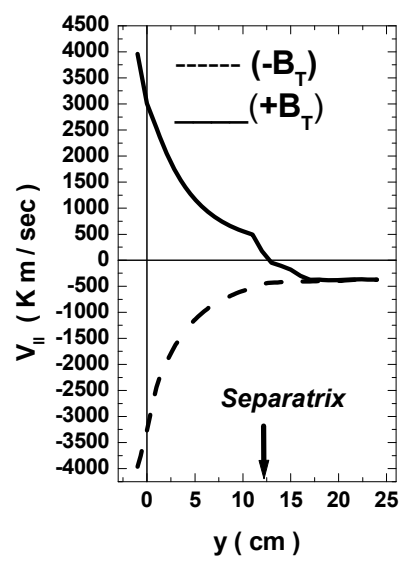

Figure 4. The radial distribution of toroidal (Parallel) velocity at normal and reverse toroidal magnetic field $B_{T}$.

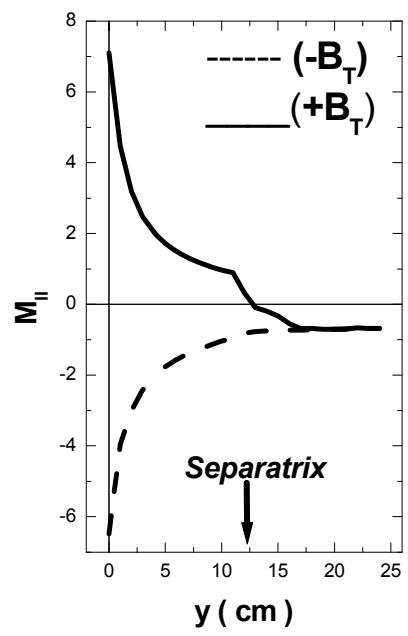

Figure 5. The radial distribution of parallel Mach number at normal and reverse toroidal magnetic field $B_{T}$.

(LFS) are shown in Figures 6, 7. In Figures 6, 7, near separatrix, the radial electric at HFS and LFS are nonmonotonic Here mainly the radial transport of parallel momentum driven by parallel viscosity balance the anomalous radial transport of parallel momentum driven by anomalous viscosity. The non-monotonic electric field is responsible for spikes near separatrix at (HFS) and (LFS). The magnitude of this spikes are dependent on plasma electrostatic potential at (HFS) and (LFS). Also the sign and magnitude of this spikes are controlled by controlled the toroidal (parallel) fluxes at (HFS) and (LFS), which transport to SOL and divertors plates through separatrix. This result agrees with the result [9].

4) The simulation result shows that, for reverse toroidal magnetic field, the strong ITB is located between the positions of maximum and minimum radial electric field shear as shown as in Figure 8. This result consistent 


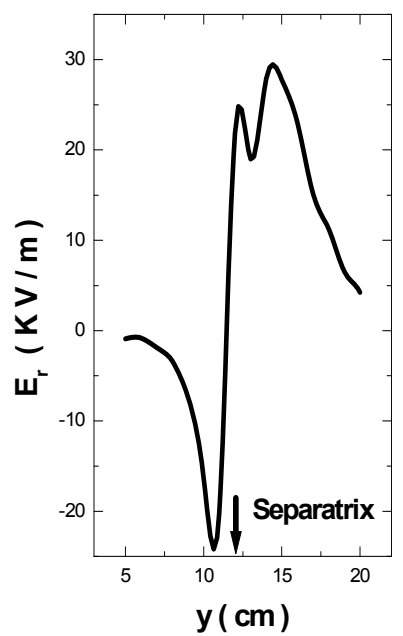

Figure 6. The radial electric field distribution at (HFS) in edge plasma of SSDT.

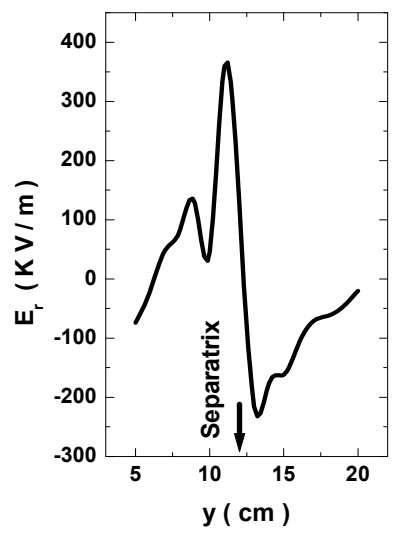

Figure 7. The radial electric field distribution at (LFS) in edge plasma of SSDT.

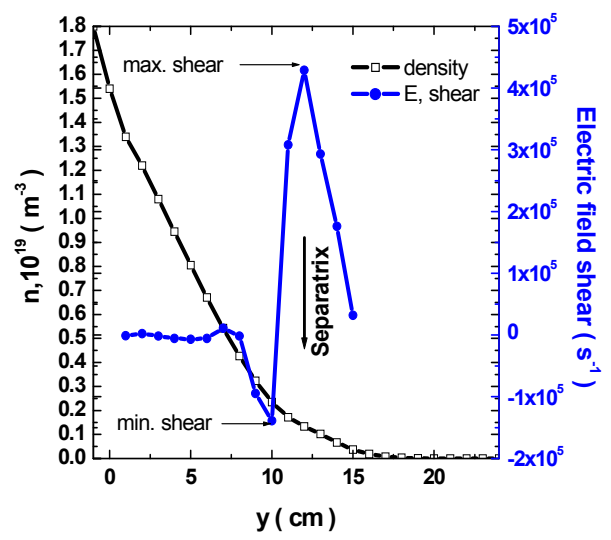

Figure 8. The radial distribution of plasma density and electric field shear at reverse toroidal magnetic field.

with the result $[10,11]$. This result is interesting since they might help explain for reversed toroidal magnetic field the easier transition to $\mathrm{H}$-regime and strong ITB formation in edge plasma of small size divertor tokamak

5) The simulation results shows that, at region of strong shear of radial electric field a large reduction of poloidal rotation was observed as shown in Figure 9. Figure 9 shows that, the poloidal flux in SOL is very weak and poloidal flux to the targets is $0.3 \%$ of total ion flux across separatrix for normal direction of toroidal magnetic, but for reverse direction of toroidal magnetic field the poloidal flux to the targets is $0.02 \%$ of total ion flux across separatrix.

6) The fifth result of simulation shows that, the reversal toroidal magnetic field make strong change in the divertor properties leading to inner divertor plate receive heat flux greater than outer divertor plate as shown in Figure 10. This result consistent with results of [6-8].

7) The sixth result of simulation shows that, the poloidal heat flux is toroidal magnetic field direction dependence as shown as in Figure 10.

\section{CONCLUSIONS}

The simulation provides the following results:

1) Parallel (toroidal) flow flux is reverse with reverse

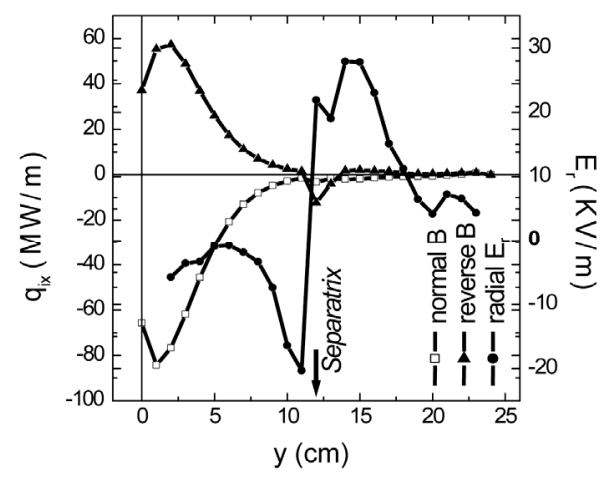

Figure 9. The radial distribution of poloidal heat flux and electric field at normal and reverse toroidal magnetic field.

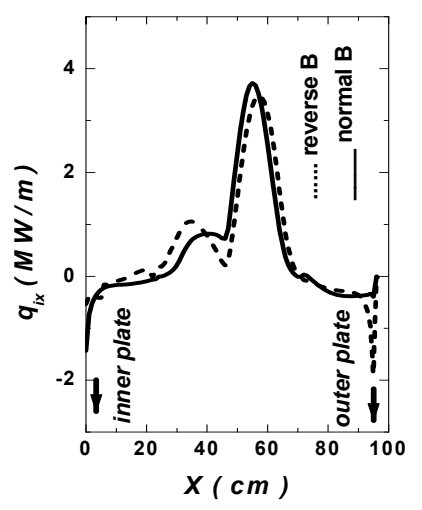

Figure 10. The poloidal distribution of poloidal heat flux at normal and reverse toroidal magnetic field. 
toroidal magnetic field, with Mach number up to 0.6.

2) The radial electric field of small size divertor tokamak (SSDT) is toroidal magnetic field independence.

3) The simulation result shows that, for reverse toroidal magnetic field, the strong ITB is located between the positions of maximum and minimum radial electric field shear. This result is interesting since they might help explain for reversed toroidal magnetic field the easier transition to H-regime and strong ITB formation in edge plasma of small size divertor tokamak.

4) Near separatrix the radial electric field at (HFS) and (LFS) are non-monotonic. The non-monotonic structure of radial electric field is connected with the radial transport of parallel (toroidal) momentum driven by parallel (toroidal) viscosity balances the anomalous radial transport driven anomalous viscosity. Also the non-monotonic structure of radial electric field is responsible for spikes formed near separatrix at HFS and LFS. The peaks of those spikes are dependent upon the plasma electrostatic potential between plasma and material walls and divertors plates. Also the sign and magnitude of this spikes are controlled by controlled the toroidal (parallel) fluxes at (HFS) and (LFS), which transport to SOL and divertors plates through separatrix.

5) The difference between the structure of the radial electric field at HFS and LFS refer to the change in parallel flux transport to SOL through separatrix. This result consistent with the result [9].

6) In the region of strong shear of radial electric field, a large reduction of poloidal rotation was observed and poloidal flux to the targets is $0.3 \%$ of total ion flux across separatrix for normal direction of toroidal magnetic, but for reverse direction of toroidal magnetic field the poloidal flux to the targets is $0.02 \%$ of total ion flux across separatrix.

7) The poloidal heat flux is toroidal magnetic field direction dependence.

8) The reversal toroidal magnetic field make strong changes in the divertor properties leading to inner divertor plate receive power flux greater than outer divertor plate. This result consistent with results of [6-8].

\section{REFERENCES}

[1] Bekheit, A.H. (2008) Simulation of small size divertor tokamak plasma edge including self consistent electric field. Jouenal of Fusion Energy, 27, 338-345.

doi:10.1007/s10894-008-9148-Z

[2] LaBombard, H., Hutchinson, B., Lipshultz, McCracken, G., Snipes J. and Terry J., (1995) The effects of field reversal on the Alcator C-Mod divertor. Journal of Plasma Physics Control Fusion, 37, 1389-1406.

doi:10.1088/0741-3335/37/12/004

[3] Chankin, A.V. Stangeby, P.C., K, Erents, P. and Harbour, J., Lingertat, (1994) The effect of $B_{T}$ reversal on the asymmetries between the strike zones in single null divertor discharges: experiment and theories. Journal of Plasma Physics Control Fusion, 37, 1853-1864. doi:10.1088/0741-3335/36/11/011

[4] Schneider, R, Rozhansky, V. and Voskoboynikov, V. (2001) Simulation of tokamak edge plasma including self-consistent electric field. Journal of Nuclear Fusion, 41, 387. doi:10.1088/0029-5515/41/4/305

[5] Murphy, S.A. (2011) Glow Discharges and Tokamaks: Principles and Application of Small Size Divertor Tokamak, Nova Science Publisher, Hauppauge.

[6] Rognlien, T.D., Ryutov, D. and Mattor, N. (1999) Influence of $\mathrm{E} \times \mathrm{B}$ and $\nabla \mathrm{B}$ drift terms in 2-D edge /SOL transport simulation. Journal of Nuclear Materials, 266269, 654-659. doi:10.1016/S0022-3115(98)00835-6

[7] Wolfe, S., LaBombard, H., Hutchinson, B., Lipshultz, G., McCracken, J., Snipes and Terry, J. (1997) Experimental investigation of transport phenomena in the Scrape Off layer and divertor. Journal of Nuclear Materials, 241-243, 149-166.

[8] Boedo, J. (1999) Convection in the DIII-D Divertor. Evolved Packet System, NewYork.

[9] Rozhansky, V., Voskoboynikov, V. and Schneider, R (2006) Modelling of radial electric field profile for different divertor configuration. Journal of Plasma Physics Control Fusion, 48, 1425-143. doi:10.1088/0741-3335/48/9/011

[10] Ide, S., Suzuki, T., Koide, Y., Takenage, H., Kamada, Y., Fujita, T. Shirai, H. and the JT-60 Team, (2004) Properties of internal transport barrier formation in JT-60U. Journal of Nuclear Fusion, 44, 876-882. doi:10.1088/0029-5515/44/8/006

[11] Bekheit, A.H. (2010) Simulation of the radial electric field shear in the edge plasma of small size divertor tokamak. Journal of Fusion Energy, 29, 267-270. doi:10.1007/s10894-009-9271-5 